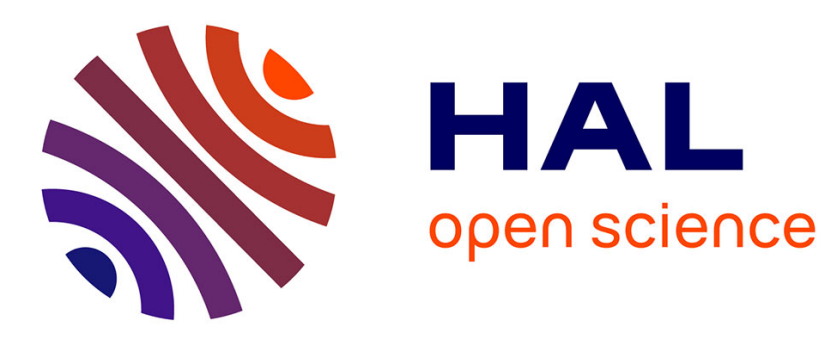

\title{
Déformation plastique de l'oxyde de nickel monocristallin
}

\author{
A. Dominguez-Rodriguez, J. Castaing
}

\section{To cite this version:}

A. Dominguez-Rodriguez, J. Castaing. Déformation plastique de l'oxyde de nickel monocristallin. Revue de Physique Appliquée, 1976, 11 (3), pp.387-391. 10.1051/rphysap:01976001103038700 . jpa00244072

\section{HAL Id: jpa-00244072 https://hal.science/jpa-00244072}

Submitted on 1 Jan 1976

HAL is a multi-disciplinary open access archive for the deposit and dissemination of scientific research documents, whether they are published or not. The documents may come from teaching and research institutions in France or abroad, or from public or private research centers.
L'archive ouverte pluridisciplinaire HAL, est destinée au dépôt et à la diffusion de documents scientifiques de niveau recherche, publiés ou non, émanant des établissements d'enseignement et de recherche français ou étrangers, des laboratoires publics ou privés. 
Classification
Physics Abstracts

7.222

\title{
DÉFORMATION PLASTIQUE DE L'OXYDE DE NICKEL MONOCRISTALLIN \\ $(*)$
}

\author{
A. DOMINGUEZ-RODRIGUEZ (**) et J. CASTAING
}

\author{
Laboratoire de Physique des Matériaux, C. N. R. S., Bellevue, 92190 Meudon (France)
}

(Reçu le 7 janvier 1976, accepté le 29 janvier 1976)

\begin{abstract}
Résumé. - Des expériences de compression à vitesse constante ont été réalisées sur des monocristaux de NiO orientés pour activer le système de glissement $\{110\}<\overline{1} 10\rangle$.

$\mathrm{La}$ contrainte d'écoulement plastique a été déterminée dans l'air, entre $20^{\circ} \mathrm{C}$ et $1200^{\circ} \mathrm{C}$ : elle subit une brusque augmentation à la température de Néel, une instabilité du type effet PortevinLe Chatelier entre $400^{\circ} \mathrm{C}$ et $900{ }^{\circ} \mathrm{C}$, et une importante diminution après recuit des éprouvettes sous faible pression d'oxygène.
\end{abstract}

\begin{abstract}
Constant strain rate experiments have been performed on NiO single crystals oriented for $\{110\}<\overline{1} 10>$ glide.

The flow-stress has been measured in air between $20^{\circ} \mathrm{C}$ and $1200^{\circ} \mathrm{C}$ : it shows a sharp increase at the Neel temperature, serrations as in the Portevin-Le Chatelier effect between $400^{\circ} \mathrm{C}$ and $900{ }^{\circ} \mathrm{C}$ and a large decrease after annealing the specimens in oxygen at low pressure.
\end{abstract}

1. Introduction. - Les oxydes constituent les matériaux de base pour beaucoup d'utilisations à haute température dans une atmosphère corrosive. Leur fragilité et leur faible résistance au choc thermique constituent une sérieuse limitation pour les applications. L'augmentation des connaissances fondamentales sur les propriétés physiques des oxydes a permis d'envisager leur utilisation dans des domaines nouveaux comme par exemple l'optique ou l'électronique. Toutefois peu d'efforts ont porté sur l'étude des mécanismes de base de la déformation plastique et de l'influence des dislocations sur les propriétés physiques des oxydes. L'objet du présent travail est de déterminer les conditions dans lesquelles l'oxyde de nickel $\mathrm{NiO}$ se déforme plastiquement, et d'étudier les mécanismes de mouvement des dislocations.

De nombreuses études de ce type ont été et sont encore faites sur des cristaux ioniques comme $\mathrm{NaCl}$ [1] et $\mathrm{MgO}$ [2]. La liaison chimique de l'oxyde de nickel également de structure $\mathrm{NaCl}$, au-dessus de la température de Néel de $520 \mathrm{~K}$, peut être décrite dans un modèle ionique bien qu'on puisse le ranger dans la catégorie des semiconducteurs [3]. L'oxyde de nickel a en outre la particularité de pouvoir présenter des écarts à la

(*) Partie de la Thèse de A. D. R. (Université de Séville, 1975), ce travail a bénéficié de l'Action Thématique Programmée du C. N. R. S. Propriétés mécaniques des Solides.

(**) Adresse actuelle : Departmento de Optica, Facultad de Ciencias, Universidad de Sevilla (Espagne). stæchiométrie (Fig. 1) que l'on explique par la présence de lacunes ionisées de nickel associées à des trous dans la bande de valence [4]; l'influence de ces défauts atomiques sur les propriétés plastiques n'est pas connue.

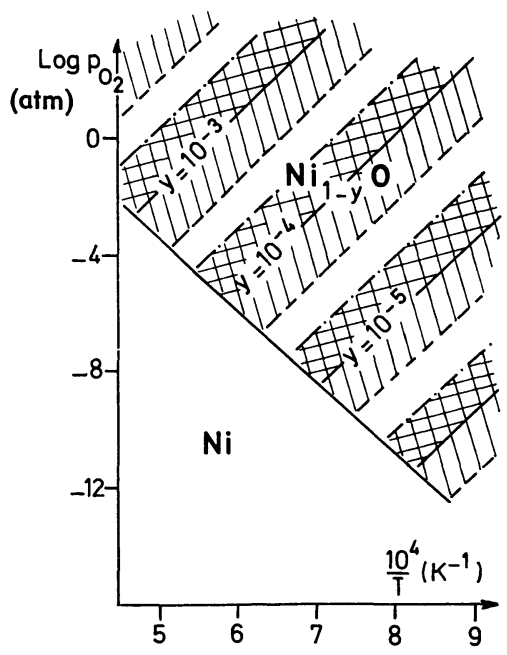

Fig. 1. - Diagramme d'équilibre pression d'oxygène-tempéra. ture de l'oxyde de nickel, établi d'après les données de la référence [4]. La zone quadrillée correspond à l'intervalle de meilleur accord des divers résultats.

Quelques études sur les propriétés mécaniques de $\mathrm{NiO}$ ont été faites en vue d'expliquer le comportement du nickel au cours de son oxydation à haute tempé- 
rature. Menzies et al. [5] [6] ont étudié les propriétés mécaniques de lames de $\mathrm{NiO}$ obtenues par oxydation totale du métal. Les essais mécaniques sont faits en flexion centrale et montrent l'apparition de la déformation plastique à partir de $850^{\circ} \mathrm{C}$. Evans et al. [7] ont réalisé des expériences de compression à vitesse constante sur des échantillons polycristallins de porosité et de pureté variables ; le matériau devient plastique entre $300^{\circ} \mathrm{C}$ et $400^{\circ} \mathrm{C}$ avec une limite élastique de 650 à $500 \mathrm{~N} / \mathrm{mm}^{2}$; et il montre un durcissement notable en présence de $5 \%$ de cobalt ou de manganèse. Tous ces résultats ne portent que sur les propriétés mécaniques macroscopiques de polycristaux; nous avons donc entrepris une étude systématique sur des monocristaux d'oxyde de nickel.

2. Techniques expérimentales. -2.1 FABriCATION DES ÉCHANTLLONS. - Des monocristaux de 2 à $3 \mathrm{~cm}$ de longueur et de 0,5 à $1 \mathrm{~cm}$ de diamètre sont obtenus par fusion de zone dans l'air dans un four image [8]. Les éprouvettes sont clivées et découpées à l'aide d'une scie à disque diamanté ; les faces découpées sont polies à la pâte diamant de $5 \mu$; on obtient ainsi des parallélépipèdes de faces $\{100\}$ et de dimensions voisines de $6 \times 2,5 \times 2,5 \mathrm{~mm}^{3}$.

Une fois découpées, les éprouvettes subissent un recuit à $1200^{\circ} \mathrm{C}$, pendant une centaine d'heures, à deux pressions partielles d'oxygène différentes qui sont l'air et $10^{-6}$ atm $(0,1 \mathrm{~Pa})$; ce traitement est destiné à modifier l'écart à la stœchiométrie de $\mathrm{NiO}$ (Fig. 1. $y \sim 10^{-3}$ et $10^{-4}$ ). Après recuit les échantillons sont trempés à l'air dans le premier cas, et refroidis rapidement dans le four dans le deuxième cas.

2.2 CARACTÉRISATION DES ÉCHANTILlons. - La poudre utilisée (oxyde de nickel pour analyses Prolabo) pour la fabrication des barreaux subissant la fusion de zone étant de pureté assez basse, les cristaux contiennent encore une certaine quantité d'impuretés ; une analyse par spectroscopie d'émission a donné comme teneur en ppM Co, 230 ; Mn, 315 ; Mg, 210 ; $\mathrm{Cr}, 40$; Ca, 45 ; Cu, 24 ; Al, 20. Nous avons cherché le meilleur traitement de surface pour l'examen par topographie par rayons $\mathrm{X}$ suivant la méthode de BergBarrett [9].

Le clivage n'introduit pas de contraintes apparentes alors qu'un polissage mécanique doit être obligatoirement suivi d'un polissage chimique pour enlever la couche écrouie; un recuit de plusieurs centaines d'heures à $1200^{\circ} \mathrm{C}$ n'est pas suffisant pour atteindre ce résultat. Nous avons donc préféré faire les observations dans les faces clivées des éprouvettes. La figure 2 montre la structure d'un échantillon : elle est constituée de sous-grains de dimension de $100 \mu \mathrm{m}$ environ.

Ce résultat est confirmé par attaque chimique également sur des faces clivées : l'immersion de $\mathrm{NiO}$ dans de l'acide nitrique concentré à $105^{\circ} \mathrm{C}$ pendant environ $30 \mathrm{mn}$ [10], permet de révéler les émergences des dislocations. La densité de dislocations à l'intérieur des

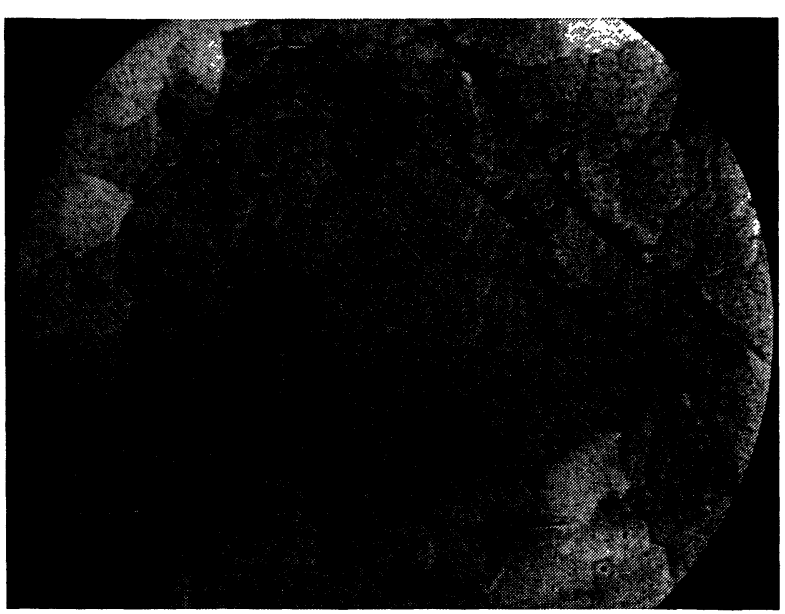

FIG. 2. - Topographie de Berg-Barrett d'une face clivée (100) de $\mathrm{NiO}$; plan réflecteur (200), divergence horizontale du faisceau $24^{\prime}$.

sous-grains, avant déformation, est de l'ordre de $10^{5} \mathrm{~cm}^{-2}$.

2.3 ESSAIS MÉCANIQUES. - Les essais mécaniques ont été réalisés dans une machine Instron équipée pour la compression directe des éprouvettes [11] ; ils ont été faits dans l'air entre la température ambiante et $1200^{\circ} \mathrm{C}$, l'équilibre thermique de l'ensemble étant atteint après une heure environ. La charge est appliquée parallèlement à la direction $<001>$ par suite du mouvement à vitesse constante (en général $20 \mu \mathrm{m} / \mathrm{mm}$ ) de la traverse de la machine Instron. La déformation de l'éprouvette est calculée à partir du déplacement de la traverse après soustraction de la déformation de la machine ; la force est mesurée avec une sensibilité de $100 \mathrm{~N}$ pleine échelle sur l'enregistreur. Les courbes contrainte-déformation tracées sont les courbes de la contrainte $\sigma=F / S_{0}$ en fonction de la déformation $\varepsilon=\left(l_{0}-l\right) / l_{0}$ où $F$ est la charge, $S_{0}$ la section initiale, $l$ la longueur et $l_{0}$ la longueur initiale de l'éprouvette.

Des essais de saut de vitesse et de relaxation ont été utilisés pour déterminer le volume d'activation $V$ du processus de déformation; dans le premier cas on utilise la relation :

$$
V=\left.2 k T \frac{\partial \ln \dot{\varepsilon}}{\partial \sigma}\right|_{T} .
$$

Dans le deuxième cas on porte $\sigma-\sigma_{0}$ en fonction de Log $t ; V$ s'obtient par la relation [12]

$$
\sigma-\sigma_{0}=\frac{2 k T}{V} \ln \frac{t+c}{c}
$$

$\dot{\varepsilon}=$ vitesse de déformation,

$\sigma=$ contrainte à l'instant $t$,

$\sigma_{0}=$ contrainte à l'instant initial $t=0$ de la relaxation

$c=$ constante, négligeable pour $t$ grand.

Le facteur 2 introduit le facteur de Schmid du système de glissement observé. 
3. Résultats des expériences. - 3.1 MORPHOLOGIE DU GLISSEMENT. - Les figures d'attaque obtenues sur des échantillons déformés montrent que le système de glissement qui se déclenche est $\{110\}<\overline{1} 10\rangle$ comme dans tous les cristaux de structure $\mathrm{NaCl}$. Quatre plans de glissement peuvent opérer; à haute température $\left(T>T_{\mathrm{N}}\right)$, et pour les échantillons de section carrée, on n'observe que deux plans orthogonaux comme l'atteste la forme en barillet après de fortes déformations. A température ambiante deux plans glissent au début, puis les deux autres commencent progressivement à opérer après 1 ou $2 \%$ de déformation et le taux de consolidation augmente (Fig. 3). Le nombre de plans de glissement dépend

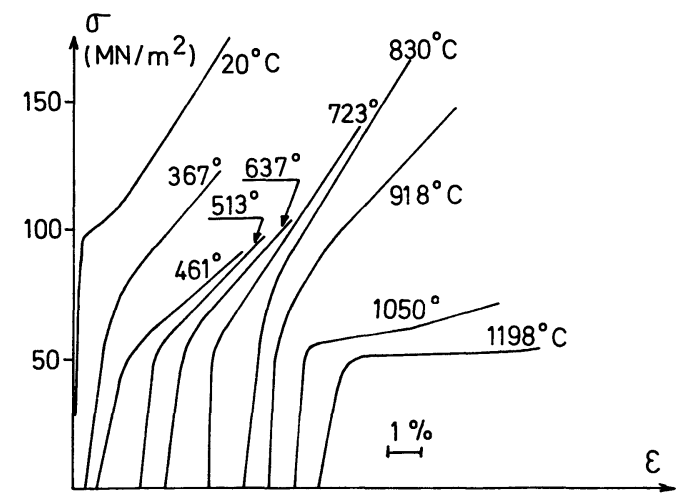

FIG. 3. - Courbes contrainte-déformation nominales de NiO pour différentes températures. Dans le domaine de température de l'effet Portevin-Le Chatelier, les hachures n'ont pas été représentées ; échantillons recuits dans l'air à $1200^{\circ} \mathrm{C}$.

également de la forme de l'éprouvette de compression. $\mathrm{Si}$ la section est rectangulaire, on n'observe que les deux plans de glissements de parcours le plus petit. Des variations dans la forme des éprouvettes entraînent une dispersion de la résistance mécanique ; les dimensions indiquées au paragraphe 2.1 minimisent cette dispersion comme dans le cas de LiF [13].

3.2 Déformation PLASTIQUe DES ÉChaNTILLONS RECUITS DANS L'AIR. - L'oxyde de nickel monocristallin a été comprimé avec une vitesse de déformation $\dot{\varepsilon}$ de l'ordre de $6 \times 10^{-5} \mathrm{~s}^{-1}$. Dès la température ambiante les cristaux se déforment plastiquement jusqu'à un taux de 6 à $7 \%$ (Fig. 3) à partir duquel apparaissent des fissures conduisant à la rupture des échantillons. Lorsque la température augmente, les cristaux peuvent subir des déformations de plus en plus grandes. La figure 3 représente les courbes contraintedéformation à diverses températures et la figure 4 représente la variation avec la température du taux de consolidation et de la contrainte d'écoulement plastique à $0,2 \%$ de déformation.

Plusieurs caractéristiques se dégagent de ces courbes :

- forte activation thermique de la contrainte d'écoulement en dessous de la température de Néel $T_{\mathbf{N}}$;

- discontinuité de la contrainte à $T_{\mathrm{N}}[14]$;

- zone athermale entre $400^{\circ} \mathrm{C}$ et $700^{\circ} \mathrm{C}$;

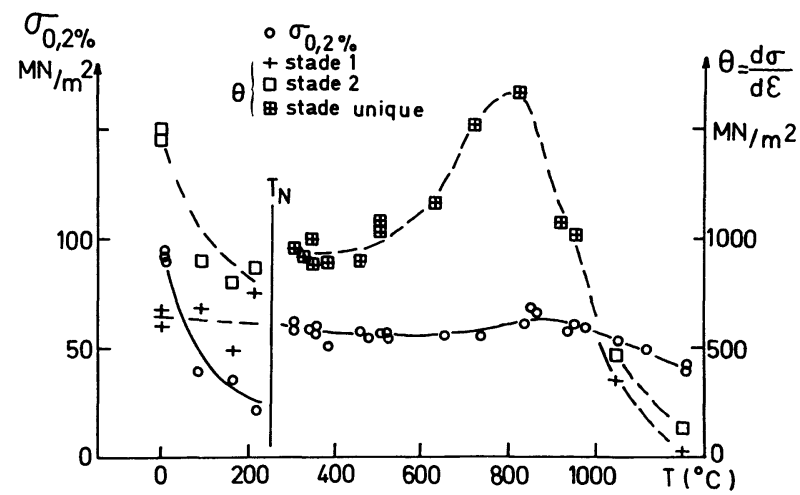

Fig. 4. - Variation avec la température de la contrainte nominale d'écoulement à $0,2 \%$ de déformation et du taux de consolidation pour NiO recuit dans l'air. Dans le cas où les courbes contrainte-déformation (Fig. 3) présentent deux stades, on a porté deux points.

- existence de deux stades dans le glissement en dessous de la température de Néel ;

- à haute température, décroissance de la contrainte et du taux de consolidation.

De plus, des hachures apparaissent sur les courbes contrainte-déformation entre $400{ }^{\circ} \mathrm{C}$ et $900^{\circ} \mathrm{C}$ et le taux apparent de consolidation passe par un maximum vers $800^{\circ} \mathrm{C}$ (Fig. 4$)$; ce phénomène peut être relié à l'effet Portevin-Le Chatelier qui a été décrit en détail ailleurs [15].

Les méthodes de relaxation de contrainte et de saut de vitesse ont été utilisées pour déterminer les volumes d'activation sauf à basse température où la fragilité des cristaux a imposé de n'utiliser que la première technique. Les essais ont été limités aux températures inférieures à $T_{\mathrm{N}}$ et supérieures à $800^{\circ} \mathrm{C}$; dans le domaine intermédiaire où apparaît l'effet Portevin-Le Chatelier, il n'y a pas de relaxation de la contrainte quand on arrête la traverse et la contrainte est insensible à la vitesse de déformation.

Les volumes d'activation ont été portés en fonction de leur contrainte de mesure dans les figures 5 et 6 . Les fortes contraintes correspondent aux essais réalisés à basse température et inversement. A l'approche de la température de Néel, les valeurs du volume d'activation sont très fortes [14]; elles n'ont pas été mesurées avec précision et les points correspondants ne sont pas sur la figure 5. A haute température, le volume d'acti-

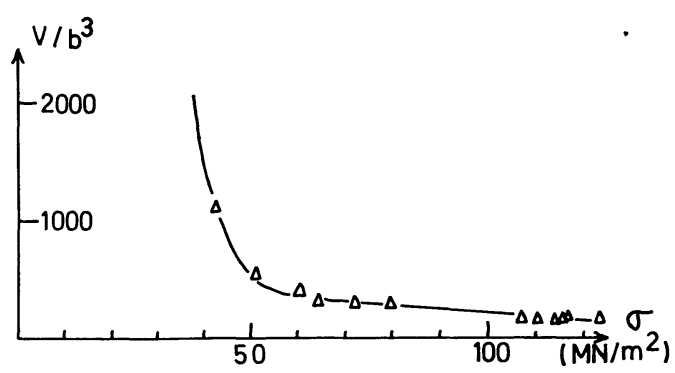

Fig. 5. - Volume d'activation en fonction de la contrainte d'écoulement plastique en dessous de la température de Néel pour $\mathrm{NiO}$ recuit dans l'air ; $b=2,96 \AA$. 


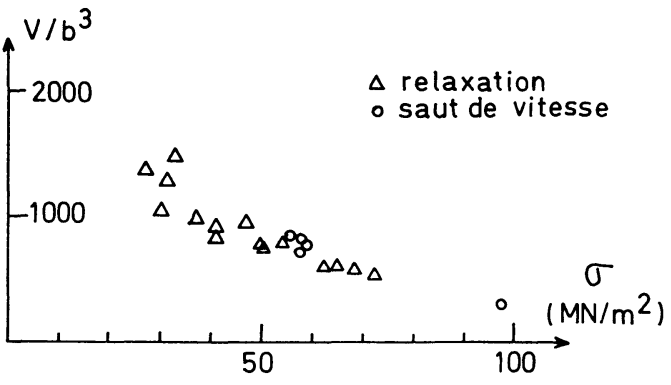

FIG. 6. - Même chose que figure 5 pour des températures , supérieures à $850^{\circ} \mathrm{C}$.

vation devient également très grand (Fig. 6). Dans les deux cas, cela traduit que le franchissement des obstacles au glissement des dislocations n'est plus activé thermiquement ; il y a changement du mécanisme de contrôle de la déformation plastique.

3.3 DÉFORMATION DES ÉCHANTILLONS RECUITS SOUS FAIBLE PRESSION D'OXYGÈNE. - Quelques essais ont été faits sur des échantillons recuits sous faible pression d'oxygène. Les courbes contrainte-déformation sont similaires à celles des échantillons recuits dans l'air, mais les contraintes d'écoulement et les taux de consolidation sont beaucoup plus faibles (Fig. 7).

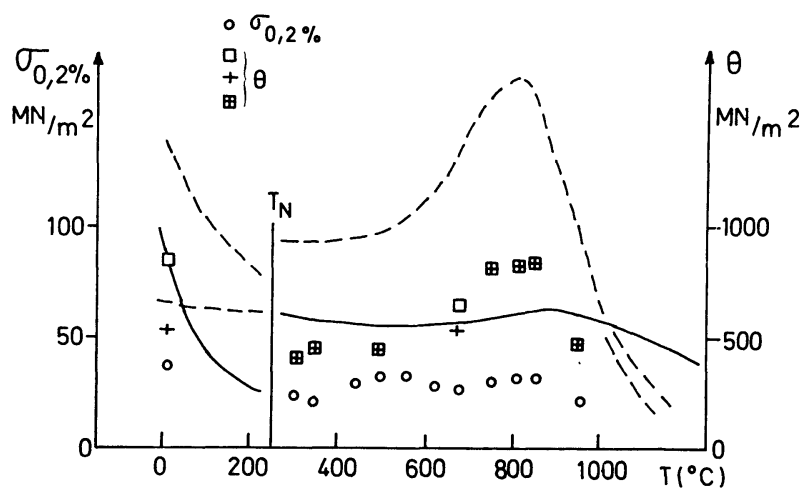

FIG. 7. - Variation avec la température de la contrainte nominale d'écoulement à $0,2 \%$ et du taux de consolidation de $\mathrm{NiO}$; échantillons recuits sous faible pression d'oxygène à $1200^{\circ} \mathrm{C}$. Les courbes tracées sur cette figure sont les courbes moyennes obtenues (Fig. 4) pour l'autre type de traitement thermique des échantillons (mêmes symboles que Fig. 4).

Les volumes d'activation déterminés à la température ambiante sont du même ordre de grandeur (environ $200 \mathrm{~b}^{3}$ ) que pour des échantillons recuits dans l'air.

Enfin, l'instabilité de l'écoulement plastique existe aussi, avec des hachures dans les courbes contraintedéformation d'amplitude analogue à celle observée pour les échantillons recuits dans l'air ; cette instabilité se manifeste également entre $450^{\circ} \mathrm{C}$ et $900^{\circ} \mathrm{C}$.

4. Discussion. - Le comportement mécanique de l'oxyde de nickel est assez proche de celui d'autres cristaux de structure $\mathrm{NaCl}$ comme par exemple $\mathrm{MgO}$; on retrouve la possibilité de déformer plastiquement des monocristaux jusqu'à la température ambiante, le durcissement par impuretés dépendant fortement du traitement thermique, c'est-à-dire de l'état des impuretés (défauts ponctuels, degré d'ionisation, association de défauts, précipités...) ; le rôle des impuretés est important pour expliquer le comportement de matériau de pureté assez faible comme celui étudié dans le présent travail.

On peut retenir, de nos expériences, les résultats suivants :

a) globalement, la contrainte d'écoulement plastique décroît lorsque la température augmente entre $20^{\circ} \mathrm{C}$ et $T_{\mathrm{N}}$ et entre $T_{\mathrm{N}}$ et $1200^{\circ} \mathrm{C}\left(1200^{\circ} \mathrm{C} \simeq 0,65 T_{\mathrm{F}}\right)$. Plusieurs plateaux athermiques se superposent à cette décroissance (Fig. 4).

b) en dessous de la température de Néel $\left(T_{\mathrm{N}} \simeq 250^{\circ} \mathrm{C}\right)$ la contrainte d'écoulement est indépendante de la température (zone athermale de 10 à $20^{\circ} \mathrm{C}$ ) et elle augmente brusquement lorsque la température dépasse $T_{\mathrm{N}}$; cette discontinuité de la contrainte n'est pas due seulement à la discontinuité du module élastique de cisaillement [14] ;

c) un autre plateau athermique de la contrainte d'écoulement apparaît vers $500^{\circ} \mathrm{C}$; il a été associé à l'effet Portevin-Le Chatelier observable dans ce domaine de température [15] ;

d) les valeurs du volume d'activation ont pu être déterminées dans des intervalles de température assez étroits; elles sont compatibles avec l'hypothèse du contrôle du glissement par l'interaction des dislocations avec les impuretés présentes dans le matériau étudié ;

- e) une très grande dépendance de la résistance mécanique avec la pression d'oxygène du recuit précédent les essais (Fig. 7) ; ce résultat pourrait être une conséquence de la variation du taux de lacunes liées à l'écart à la stœchiométrie. Il semble plus probable qu'il soit une conséquence de la variation du taux d'ionisation de certaines impuretés assez abondantes comme le manganèse ; un tel effet a également été observé dans $\mathrm{MgO}$ dopé avec du fer [16].

L'ensemble de ces résultats est assez complexes ; le nombre de paramètres expérimentaux est grand et les observations microstructurales font défaut. Une discussion plus détaillée nécessite une série d'expériences complémentaires sur des matériaux plus purs, sur des matériaux dopés avec des concentrations d'impuretés variables et connues, et également par une caractérisation précise tant des défauts responsables de la déformation (observation des dislocations par microscopie électronique en transmission) que de la nature des obstacles au glissement (impuretés...). Des travaux sont en cours dans toutes ces directions afin de permettre d'expliquer les phénomènes mis en évidence au cours de cette étude.

Remerciements. - Les auteurs remercient E. Fries et $\mathbf{J}$. Philibert pour leurs contributions à ce travail. 


\section{Bibliographie}

[1] APPel, F., Phys. Stat. Sol. (a) 25 (1974) 607.

[2] Srinivasan, M. et Stoebe, T. G., J. Mater. Sci. 9 (1974) 121.

[3] Adler, D., Solid State Phys. (Ed. F. Seitz, D. Turnbull et H. Ehrenreich, Academic Press) 1968, vol. 21.

[4] Deren, J. et Mrowec, S., J. Mater. Sci. 8 (1973) 545.

[5] Menzies, I. A. et Alred, P., Corros. Sci. 8 (1968) 525.

[6] Menzies, I. A. et Strafford, K. N., J. Mater. Sci. 2 (1967) 358.

[7] Evans, A. G., Dilip Rajdev et Douglas, D. L., Oxid. of Met. 4 (1972) 151.

[8] Saurat, M. et Revcolevschi, A., Rev. Int. Hautes Temp. Refract. 8(1971) 291.

[9] NeWKIRK, J. B., Trans. Met. Soc. A. I. M. E. 215 (1959) 483.
[10] TAKeda, T. et KondoH, H., J. Phys. Soc. Japan 17 (1962) 1315.

[11] Cadoz, J. L., Castaing, J., Dolin, C., Gervais, H. et PellisSIER, B., Revue Phys. Appl. 10 (1975) 247.

[12] Gagnon, M., Thèse, Université de Paris-Sud, Orsay, 1973.

[13] Fotedar, H. L., Srinivasan, M., Wilson, D. A. et Stoebe, T. G., Mater. Sci. Eng. 7 (1971) 272.

[14] Dominguez-Rodriguez, A. et Castaing, J., C. R. Hebd. Séan. Acad. Sci. 280 (1975) B 317.

[15] Dominguez-Rodriguez, A. et CAstaing, J., Scripta Metall. 9 (1975) 551.

[16] Moon, R. L. et Pratt, P. L., Proc. Brit. Ceram. Soc. 15 (1970) 203. 